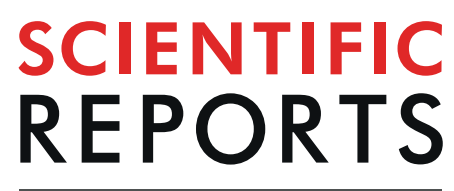

natureresearch

\title{
Crystal structure of the catalytic unit of GH 87-type $\alpha$-1,3-glucanase Agl-KA from Bacillus circulans
}

\author{
Shigekazu Yano ${ }^{1}$, Wasana Suyotha ${ }^{2}$, Natsuki Oguro일 ${ }^{1}$ Takashi Matsui ${ }^{3}{ }^{3}$, Shota Shiga ${ }^{1}$, \\ Takafumi Itoh ${ }^{4}$, Takao Hibi ${ }^{4}$, Yoshikazu Tanaka $\mathbb{1}^{3}$, Mamoru Wakayama ${ }^{5}$ \& Koki Makabe $\mathbb{C}^{1 *}$ \\ Glycoside hydrolase (GH) 87-type $\alpha$-1,3-glucanase hydrolyses the $\alpha$-1,3-glucoside linkages of \\ $\alpha-1,3$-glucan, which is found in fungal cell walls and extracellular polysaccharides produced by oral \\ Streptococci. In this study, we report on the molecular structure of the catalytic unit of GH 87-type \\ $\alpha-1,3-$ glucanase, Agl-KA, from Bacillus circulans, as determined by $x$-ray crystallography at a resolution \\ of $1.82 \AA$. The catalytic unit constitutes a complex structure of two tandemly connected domains-the \\ $\mathrm{N}$-terminal galactose-binding-like domain and the $\mathrm{C}$-terminal right-handed $\beta$-helix domain. While the \\ $\beta$-helix domain is widely found among polysaccharide-processing enzymes, complex formation with \\ the galactose-binding-like domain was observed for the first time. Biochemical assays showed that \\ Asp1067, Asp1090 and Asp1091 are important for catalysis, and these residues are indeed located at the \\ putative substrate-binding cleft, which forms a closed end and explains the product specificity.
}

$\alpha-1,3$-Glucanase (E.C.3.2.1.59) hydrolyses the $\alpha$-1,3-glucoside linkages in $\alpha-1,3$-glucan, which is found in fungal cell walls and extracellular polysaccharides produced by oral Streptococci ${ }^{1,2} \cdot \alpha-1,3$-Glucanases are classified into family 71 and 87 of glycoside hydrolases (GHs) based on their amino acid sequences. GH 71-type $\alpha$-1,3-glucanases are found in fungi ${ }^{3-6}$, while GH 87 -type enzymes are found in bacteria ${ }^{7-9}$. GH 87 -type $\alpha-1,3$-glucanase was isolated from the culture filtrate of Bacillus circulans KA-304 and termed Agl-KA. Agl-KA hydrolyses the $\alpha-1,3$-glucan of oral Streptococci as well as of fungi ${ }^{10}$. Accordingly, Agl-KA has been investigated for preparing fungal protoplasts, for the analysis of $\alpha$-1,3-glucan in fungal cell walls and for the biological control of pathogenic fungi ${ }^{11,12}$.

Most GH 87 enzymes are considered multidomain enzymes. Agl-KA is also multimodular, comprising an $\mathrm{N}$-terminal discoidin domain (DS1), followed by a carbohydrate-binding module 6 (CB6), threonine and proline repeats (TP), a second discoidin domain (DS2), an uncharacterised conserved domain (UCD) and a C-terminal catalytic unit (Agl $\triangle$ DCD-UCD; Fig. 1a) $)^{13}$. The comparison of amino acid sequences of GH 87 enzymes suggests that these domains are conserved in GH 87-type $\alpha$-1,3-glucanases. In our previous study, we found that DS1, CB6 and DS2 are involved in $\alpha$-1,3-glucan binding and that these domains enhance the $\alpha$-1,3-glucan hydrolysing activity of the catalytic unit ${ }^{14}$. Furthermore, we reported that the deletion of UCD from Agl-KA does not affect $\alpha$-1,3-glucan binding activity, $\alpha-1,3$-glucan hydrolysing activity or fungal cell wall lytic activity. While these findings have revealed the functions of the individual domains of GH $87 \alpha-1,3$-glucanase, the molecular enzymatic mechanisms based on the three-dimensional structure remain unknown.

In this study, we successfully determined the x-ray crystal structure of Agl $\triangle D C D$-UCD using the Se-MAD method. We further discussed the product specificity using a combination of biochemical analysis and docking simulation.

\footnotetext{
${ }^{1}$ Department of Biochemical Engineering, Graduate School of Sciences and Engineering, Yamagata University, Jonan, Yonezawa, Yamagata, 992-8510, Japan. ${ }^{2}$ Department of Industrial Biotechnology, Faculty of Agro-industry, Prince of Songkla University, HatYai, 90112, Thailand. ${ }^{3}$ Graduate School of Life Sciences, Tohoku University, Sendai, 980-8577, Japan. ${ }^{4}$ Department of Bioscience and Biotechnology, Faculty of Biotechnology, Fukui Prefectural University, Eiheiji-cho, Yoshida-gun, Fukui, 910-1195, Japan. ${ }^{5}$ Department of Biotechnology, Faculty of Life Sciences, Ritsumeikan University, Kusatsu, Shiga, 525-8577, Japan. *email: makabe@yz.yamagata-u.ac.jp
} 
(a) 1

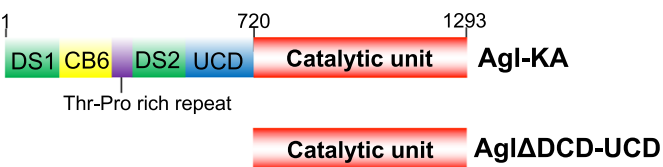

(b)

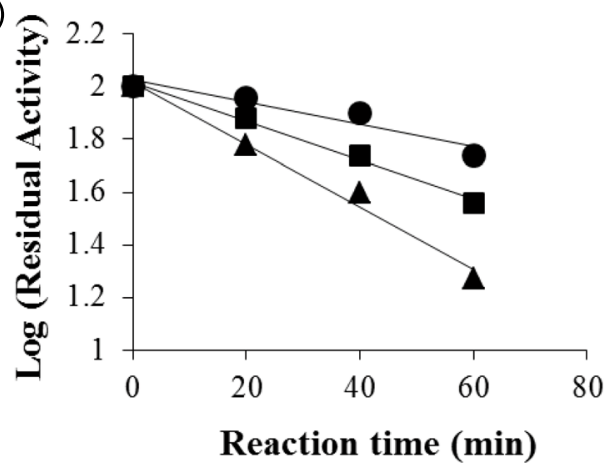

Figure 1. (a) Domain structure of Agl-KA. DS: Discoidin domain (green), CB6: Carbohydrate-binding module 6 (yellow), UCD: Uncharacterised domain (blue). Agl $\triangle$ DCD-UCD: the catalytic unit of Agl-KA (red). (b) Residual activity of Agl $\triangle \mathrm{DCD}-\mathrm{UCD}$ after chemical modification with the concentrations of $25 \mathrm{mM}(\bullet), 50 \mathrm{mM}$ ) and $100 \mathrm{mM}(\mathbf{\Delta})$ EDC.

\section{Results and Discussion}

Chemical modification of carboxyl groups in the catalytic unit of Agl-KA. To reveal amino acids essential for $\alpha$-1,3-glucan hydrolysis, Agl $\triangle \mathrm{DCD}-\mathrm{UCD}$-the catalytic unit of Agl-KA-was treated with 1-ethyl3-(3-dimethyl amino propyl) carbodimide (EDC), a chemical modifier of carboxylic amino acids used for the inhibition of enzymatic reactions. Enzyme hydrolysis decreased with incubation time and EDC concentration; after incubation for $60 \mathrm{~min}$ with $100 \mathrm{mM}$ EDC, the activity was approximately $18 \%$ of the initial activity (Fig. 1b; $10^{1.25}=18 \%$ ). These results suggest that at least one carboxylic amino acid residue is involved in catalysis and/or the substrate binding of $\alpha-1,3$-glucanase.

Mutational analysis of key residues for catalysis. Multiple alignments of the amino acid sequences of the catalytic unit from several GH $87 \alpha$-1,3-glucanases indicated that 11 carboxylic residues at positions equivalent to D737, D739, E763, D853, E854, D884, E889, E1032, D1067, D1090 and D1091 of Agl-KA were conserved among $\alpha$-1,3-glucanases (Supplementary Fig. S1). These 11 carboxylic amino acid residues were substituted with alanine to further clarify the amino acids involved in Agl-KA catalysis.

Mutant enzymes were expressed in E. coli, and resultant mutant proteins were purified from the soluble fraction of $E$. coli according to the purification method for wild-type Agl $\triangle D C D-U C D{ }^{13}$. Final purity was confirmed using SDS-PAGE (Supplementary Fig. S2). The relative activities of the wild-type and mutant enzymes are shown in Table 1. The mutants of D737A, D739A, D853A, E854A and E1032A retained approximately $80 \%-100 \%$ of their wild-type activity, indicating that the substitution of these residues had little effect on $\alpha-1,3$-glucanase activity. Activities of E763A and E889A considerably decreased; however, they remained at approximately $30 \%-40 \%$ of their wild-type activity. In contrast, the activities of D1067A, D1090A and D1091A were not detected after 30 min of incubation. After $18 \mathrm{~h}$ of incubation, D1067A, D1090A and D1091A showed only $0.09 \%, 2.84 \%$ and 3.12\% of their wild-type activity, respectively. These findings suggest that D1067, D1090 and D1091 are important for Agl-KA catalysis.

Subsequently, D1067, D1090 and D1091 were individually substituted with asparagine or glutamic acid. The activities of D1067N, D1090N and D1091N were only hardly detectable even after the extended incubation for $18 \mathrm{~h}$ (Table 1). In the glutamic acid substitution mutants, the activities of D1067E, D1090E and D1091E were $4.36 \%, 5.99 \%$ and $21.89 \%$ of their wild-type activity after $30 \mathrm{~min}$ incubation, respectively. The kinetic parameters of D1067E, D1090E, D1091E and wild-type were obtained from Lineweaver-Burk plots (Supplementary Table S2). The $K_{\mathrm{m}}$ values for D1067E, D1090E, D1091E and wild-type were approximately 42.1, 35.4, 72.4 and $36.2 \mathrm{mg} / \mathrm{mL}$, respectively. The turnover numbers $\left(k_{\text {cat }}\right)$ of D1067E, D1090E, D1091E and wild-type were approximately 6.1, 6.4, 49.7 and $81.5 \mathrm{sec}^{-1}$, respectively. The $k_{\text {cat }}$ values of D1067E and D1090E were approximately 13.5-fold lower than that of wild-type; however, the apparent $K_{\mathrm{m}}$ values were similar to that of wild-type. In contrast, the $k_{\text {cat }}$ value of D1091E was approximately 2 -fold lower and the $K_{\mathrm{m}}$ value was approximately 2 -fold higher than that of wild-type. The results of kinetic analysis indicate that D1067 and D1090 may be involved in Agl-KA catalysis and D1091 may be important for substrate binding.

Next, far-UV circular dichroism (CD) spectra of the D1067, D1090 and D1091 substituted mutants were obtained to confirm whether the overall secondary structures of enzymes were maintained. Figure 2 shows the CD spectra of the mutants and wild-type measured at the far-UV range $(200-280 \mathrm{~nm})$. All mutants showed CD spectra similar to that of wild-type, indicating that amino acid substitution did not significantly affect their secondary structure. 


\begin{tabular}{|l|l|l|}
\hline \multirow{2}{*}{ Mutation sites } & \multicolumn{3}{|l|}{ Relative activity $\mathbf{( \% )}$} \\
\cline { 2 - 3 } & $\begin{array}{l}\text { Reaction time } \\
\mathbf{3 0} \text { min }\end{array}$ & $\begin{array}{l}\text { Reaction time } \\
\mathbf{1 8 h}\end{array}$ \\
\hline Wild-type (Agl $\triangle$ DCD-UCD) & 100.0 & 100.0 \\
\hline D737A & 88.8 & 109.2 \\
\hline D739A & 100.3 & 106.2 \\
\hline E763A & 28.8 & 45.3 \\
\hline D853A & 80.8 & 86.9 \\
\hline E854A & 92.4 & 98.8 \\
\hline D884A & 80.4 & 79.5 \\
\hline E889A & 39.4 & 63.1 \\
\hline E1032A & 96.3 & 85.3 \\
\hline D1067A & n.d. & 0.09 \\
\hline D1090A & n.d. & 2.84 \\
\hline D1091A & n.d. & 3.12 \\
\hline D1067E & 4.36 & 41.0 \\
\hline D1090E & 5.99 & 36.7 \\
\hline D1091E & 21.9 & 45.4 \\
\hline D1067N & n.d. & 1.96 \\
\hline D1090N & n.d. & 0.12 \\
\hline D1091N & n.d. & 0.07 \\
\hline
\end{tabular}

Table 1. $\alpha-1,3-$ Glucanase activity of mutant enzymes.

(a)

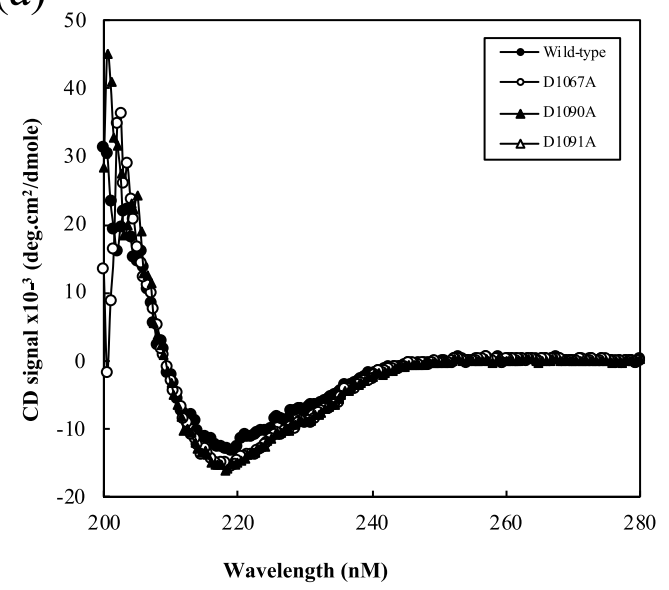

(c)

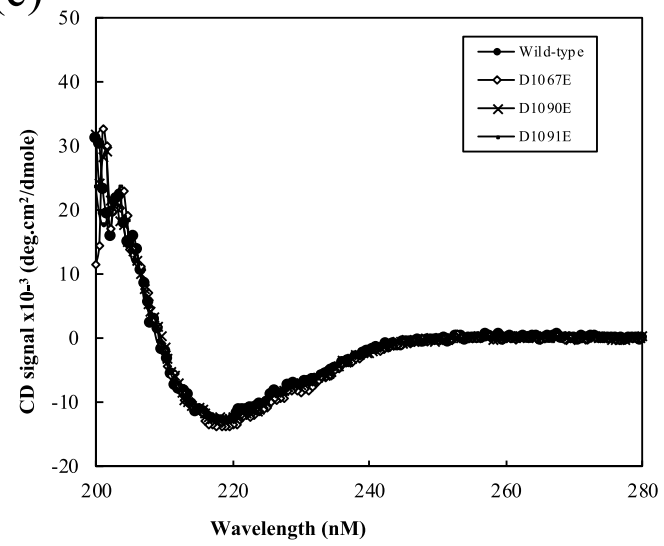

(b)

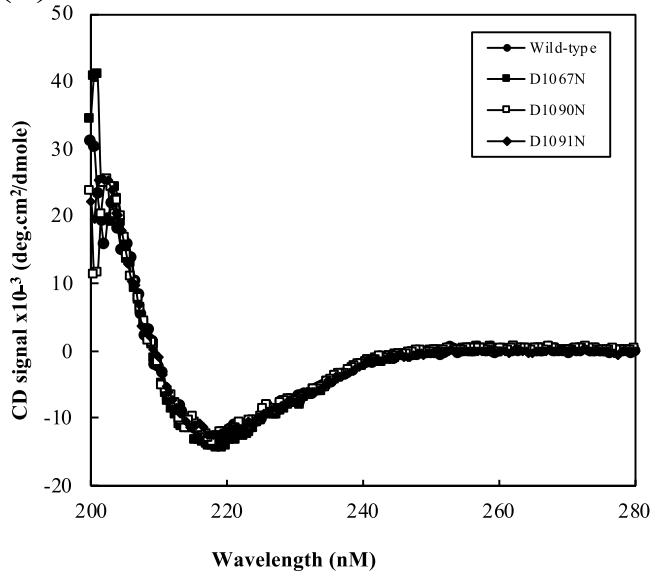

Figure 2. CD spectra of Agl $\triangle \mathrm{DCD}-\mathrm{UCD}$ with alanine (a), asparagine (b) and glutamic acid (c) substitution mutants. Abbreviations: ๑, wild-type: O, D1067A: A, D1090A: $\Delta$, D1091A:, D1067N: $\square$, D1090N: D1901N, $\oslash$, D1067E: x, D1090E: —, D1091E. 
(a)

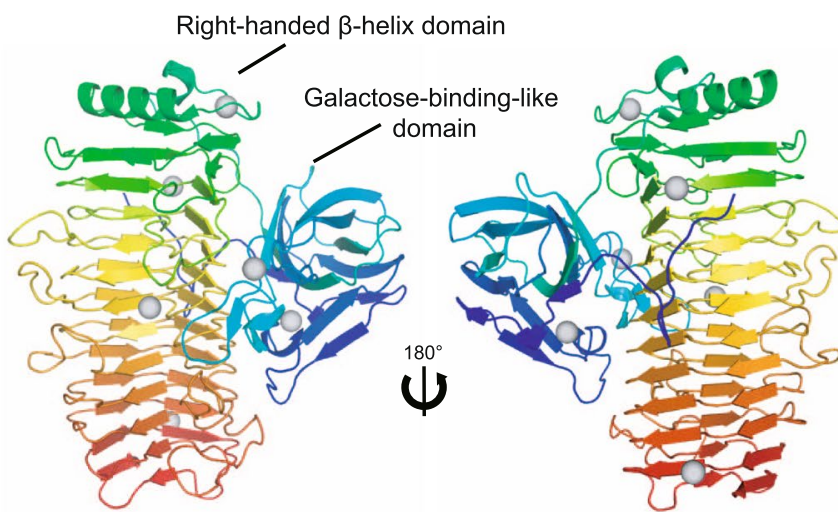

(b)

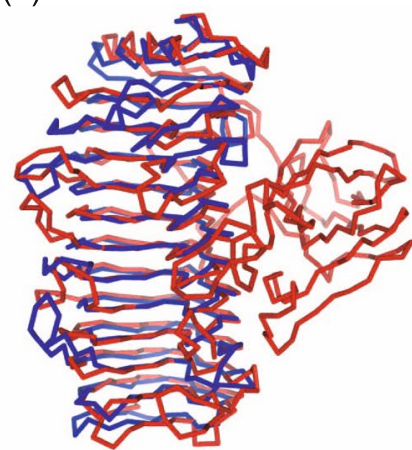

(d)

(c) Side-view
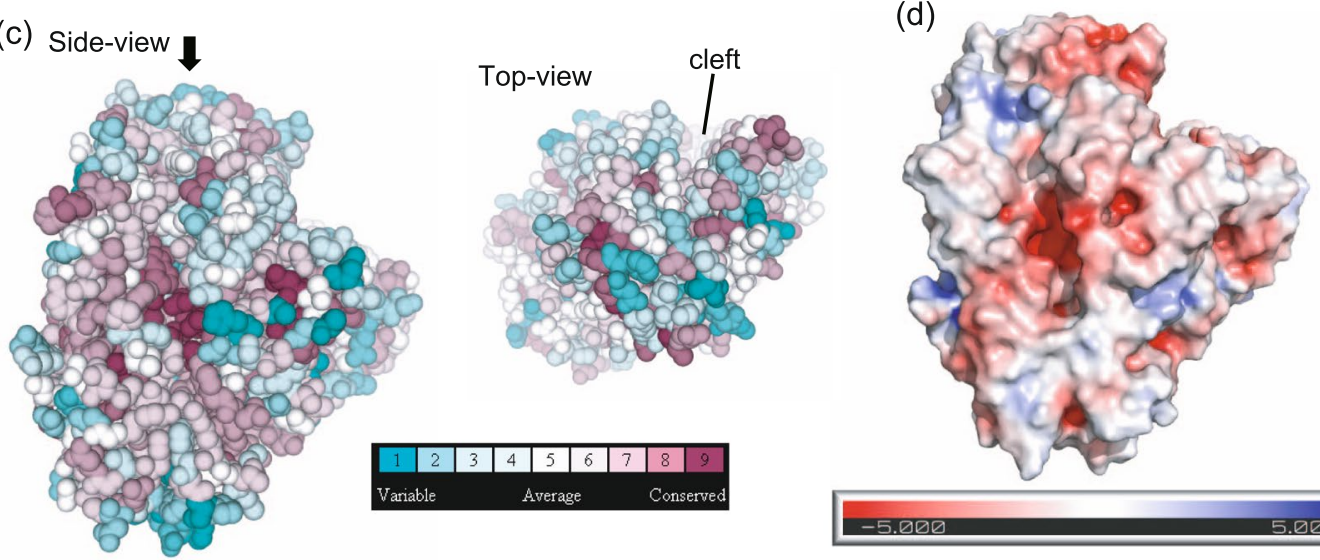

(e)

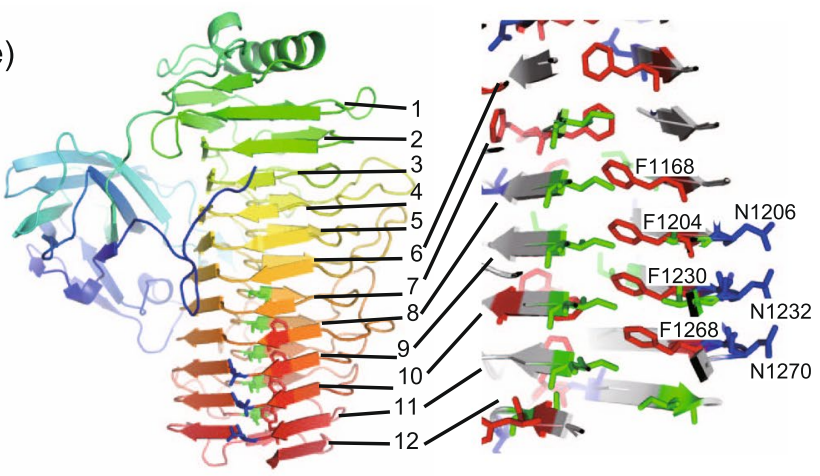

Figure 3. Crystal structure of Agl $\triangle D C D-U C D$ (PDBID: 5ZRU). (a) Ribbon representation of the structure coloured with the amino acid sequence from the N- (blue) to C-terminals (red). Six $\mathrm{Ca}^{2+}$ ions are shown as grey spheres. (b) Structure comparison with a similar structure protein, a catalytic A-module of a mannuronan C-5epimerase (AlgE4A) structure (PDBID: 2PYH; blue), from Dali server is shown with Agl $\triangle \mathrm{DCD}-\mathrm{UCD}$ (red). (c) Amino acid conservation is shown with colour as indicated. The direction of the top view is shown with a black arrow. A putative substrate-binding cleft is indicated by the top view. (d) Electrostatic potential of the molecular surface of Agl $\triangle \mathrm{DCD}-\mathrm{UCD}$ obtained using the APBS tool. The negative potential is shown in red, and the positive potential is shown in blue. (e) The twelve $\beta$-helix coils. The phenylalanine (red), asparagine (blue) and isoleucine (green) ladders are shown with stick representation.

Crystal structure of Agl $\triangle D C D-U C D$. The crystal structure of Agl $\triangle D C D-U C D$ was determined at a resolution limit of $1.83 \AA$ (Fig. 3). The crystal belonged to the space group of $\mathrm{P} 2_{1}$, and the structure was refined to the R-values of $14.1 \%$ and $17.1 \%$ for $R$ and $\mathrm{R}_{\text {free }}$, respectively (Table 2 ). Two molecules (molecules A and B) were positioned in the asymmetric unit and were essentially identical (root mean square deviation, $0.14 \AA$ ); hereafter, molecule A is used for discussion. All 575 amino acids of Agl $\triangle \mathrm{DCD}-\mathrm{UCD}$ were clearly resolved in the crystal structure, with the exception of the cloning artefact of the $\mathrm{N}$-terminus Met-Ser sequence. Five $\mathrm{Ca}^{+}$ions, two $\mathrm{Zn}^{2+}$ ions, two $\mathrm{SO}_{4} 2^{-}$molecules and one polyethylene glycol chain were observed within one protein molecule. $\mathrm{Zn}^{2+}$ ions, $\mathrm{SO}_{4}^{2-}$ molecules and polyethylene glycol were contained in the crystallisation buffer, and these molecules were localised to the protein surface; thus, we concluded that these were introduced during crystallisation. All $\mathrm{Zn}^{2+}$ ions were positioned at the interfaces of the protein molecules, contributing to the crystal packing. 


\begin{tabular}{|c|c|c|c|c|}
\hline & Native & \multicolumn{3}{|l|}{ SeMet } \\
\hline \multirow{2}{*}{\multicolumn{5}{|c|}{$\begin{array}{l}\text { Space group } \\
\text { Data collection statistics }\end{array}$}} \\
\hline & & & & \\
\hline \multirow[t]{4}{*}{ Cell parameters } & $\mathrm{a}=57.25$ & & & \\
\hline & $\mathrm{b}=126.42$ & & & \\
\hline & $c=80.54$ & & & \\
\hline & $\gamma=97.83$ & & & \\
\hline \multirow{2}{*}{ Beamline } & \multirow{2}{*}{ PF-BL5A } & \multicolumn{3}{|l|}{ PF-BL5A } \\
\hline & & Peak & Edge & Remote \\
\hline Wavelength $(\AA)$ & 1.0000 & 0.97911 & 0.97922 & 0.9640 \\
\hline Resolution $(\AA)^{\mathrm{a})}$ & $20.0-1.83(1.86-1.83)$ & \multicolumn{3}{|c|}{$49.7-2.00(2.12-2.00)$} \\
\hline Completeness (\%) & $99.4(95.5)$ & $99.8(99.3)$ & $99.5(98.5)$ & $99.5(98.5)$ \\
\hline $\mathrm{I} / \sigma(\mathrm{I})$ & $13.29(3.00)$ & $8.15(2.24)$ & $5.46(1.36)$ & $5.30(1.35)$ \\
\hline $\mathrm{R}_{\text {merge }}{ }^{b)}$ & $0.050(0.347)$ & $0.206(0.856)$ & $0.204(0.886)$ & $0.212(0.897)$ \\
\hline Average redundancy & $3.4(3.4)$ & $7.0(7.0)$ & $3.49(3.47)$ & $3.49(3.48)$ \\
\hline \multicolumn{5}{|l|}{ Refinement statistics } \\
\hline Resolution range $(\AA)$ & $19.9-1.83$ & & & \\
\hline Reflections used (free) & $98636(4962)$ & & & \\
\hline $\mathrm{R}$ factor ${ }^{\mathrm{c})}$ & 0.141 & & & \\
\hline $\mathrm{R}_{\text {free }}{ }^{\mathrm{d})}$ & 0.171 & & & \\
\hline \multicolumn{5}{|l|}{ RMS deviations } \\
\hline Bonds $(\AA ̊)$ & 0.006 & & & \\
\hline Angles $\left({ }^{\circ}\right)$ & 0.846 & & & \\
\hline Average B factor $\left(\AA^{2}\right)$ & 15.0 & & & \\
\hline \multicolumn{5}{|c|}{ Ramachandran plot statistics } \\
\hline Most favoured (\%) & 96 & & & \\
\hline Generously allowed (\%) & 4 & & & \\
\hline Disallowed (\%) & 0 & & & \\
\hline
\end{tabular}

Table 2. Data collection and refinement statistics for Agl $\triangle$ DCD-UCD (PDBID: 5ZRU). ${ }^{\text {a) }}$ The highest resolution shell is shown in parenthesis. ${ }^{\mathrm{b}} \mathrm{R}$ merge $=\Sigma_{\mathrm{hkl}} \Sigma_{\mathrm{i}}\left|\mathrm{I}(\mathrm{hkl})_{\mathrm{i}}-\langle\mathrm{I}(\mathrm{hkl})\rangle\right| / \Sigma_{\mathrm{hkl}} \Sigma_{\mathrm{i}}\left\langle\mathrm{I}(\mathrm{hkl})_{\mathrm{I}}\right\rangle$ over $i$ observations of a reflection hkl. ${ }^{c}$ R factor $=\Sigma|| F($ obs $)|-| F($ calc $)|| / \Sigma \mid F($ obs $\left.) \mid .{ }^{d}\right)$ Rfree is $\mathrm{R}$ with $5 \%$ of reflections sequestered before refinement.

The positions of three $\mathrm{Ca}^{+}$ions (out of five) were relatively buried in the protein structure, indicating these may contribute to protein stability (Fig. 3a). Consistent with this, inductively coupled plasma-mass spectrometry (ICP-MS) showed that nearly $40 \mathrm{nmol} \mathrm{Ca}{ }^{2+}$ ions per $10 \mathrm{nmol}$ protein molecules were contained in purified $\mathrm{Agl} \triangle \mathrm{DCD}-\mathrm{UCD}$ solution, whereas no $\mathrm{Zn}^{2+}$ ions were detected.

The overall structure of Agl $\triangle \mathrm{DCD}-\mathrm{UCD}$ comprised an N-terminal galactose-binding-like domain $(\sim 180$ residues), followed by a right-handed $\beta$-helix ( $\sim 400$ residues), as determined based on the CATH domain classification $^{15}$. This is consistent with the prediction by the InterPro protein sequence classification server ${ }^{16}$ (Fig. 3a). According to the structural alignment search server, Dali, the catalytic A-module of the mannuronan C-5-epimerase (AlgE4A) structure (PDBID: 2PYH), showed the highest Z-score of 37.4 and a sequence homology of $18 \%$. Though the AlgE4A structure appeared to have a similar right-handed $\beta$-helix fold, it lacked the galactose-binding-like domain, highlighting the unique entire structure of Agl $\triangle \mathrm{DCD}-\mathrm{UCD}$ (Fig. 3b). In addition to the two domains, there appeared to be an N-terminal extension before the galactose-binding-like domain ( $\sim 14$ residues) as well as a linker between the two domains ( $\sim 11$ residues). These regions were resolved with clear electron density maps (Supplementary Fig. S3a,b). The entire structure of Agl $\triangle D C D$-UCD was $\beta$-sheet rich and consistent with the observed CD spectra (Fig. 2a). The right-handed $\beta$-helix fold is a common structural class for carbohydrate-binding proteins called "CASH (arbohydrate-binding proteins and sugar hydrolase)" ${ }^{17}$, and the C-terminal catalytic unit of the GH 87 family was also predicted to share this common fold ${ }^{18}$.

The striking difference between Agl $\triangle \mathrm{DCD}-\mathrm{UCD}$ and other $\beta$-helix folds of CASH is the presence of the galactose-binding-like domain, which forms a complex with the $\beta$-helix domain (Fig. 3a). The interface area between the galactose-binding-like domain and the $\beta$-helix domain was $1743 \AA 2$, indicating that a large surface area was buried upon complex formation and the complex was stabilised. The amino acid sequence of the galactose-binding-like domain is highly conserved among the GH 87 family (Supplementary Fig. S1), indicating that this complex structure is a common structural feature in this family. Figure $3 c$ shows the conserved amino acids in the GH 87 family; highly conserved residues are located at the bottom of the cleft, which is a putative active site. Amino acids at the interface between the galactose-binding-like domain and the $\beta$-helix domain are also conserved (Supplementary Fig. S3c). Figure 3d shows the electrostatic surface potential and putative active site at the conserved substrate-binding cleft with negative surface potential.

Although the structure of Dex49, a GH 49 family dextranase, shows a similar complex structure comprising an $\mathrm{N}$-terminal $\beta$-sandwich domain and a $\beta$-helix domain $^{19}$, the $\beta$-sandwich domain shares no sequence homology 

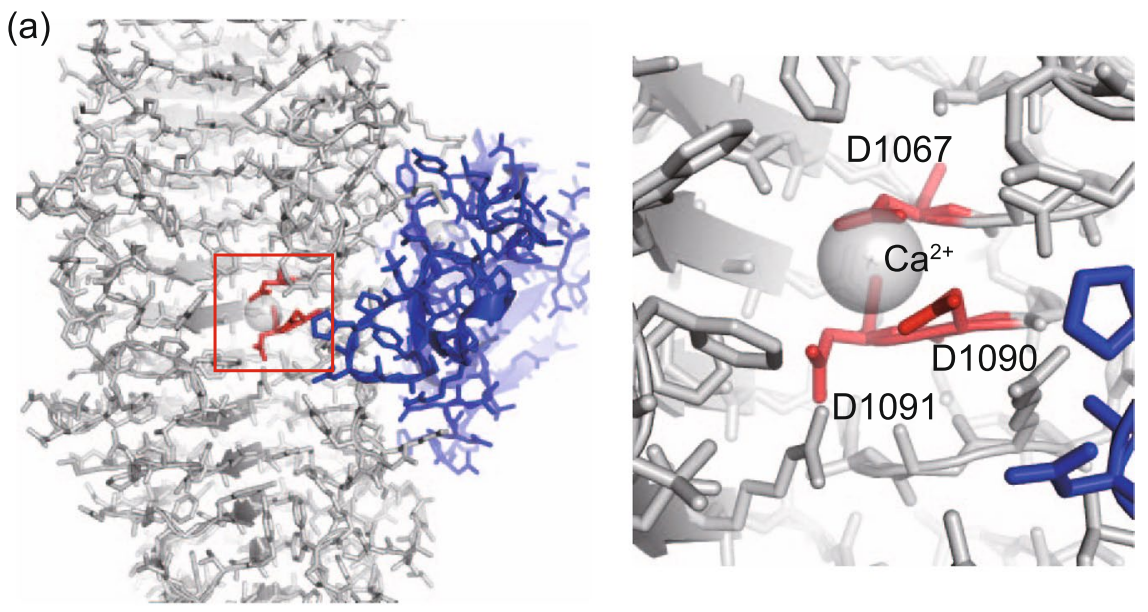

(b)

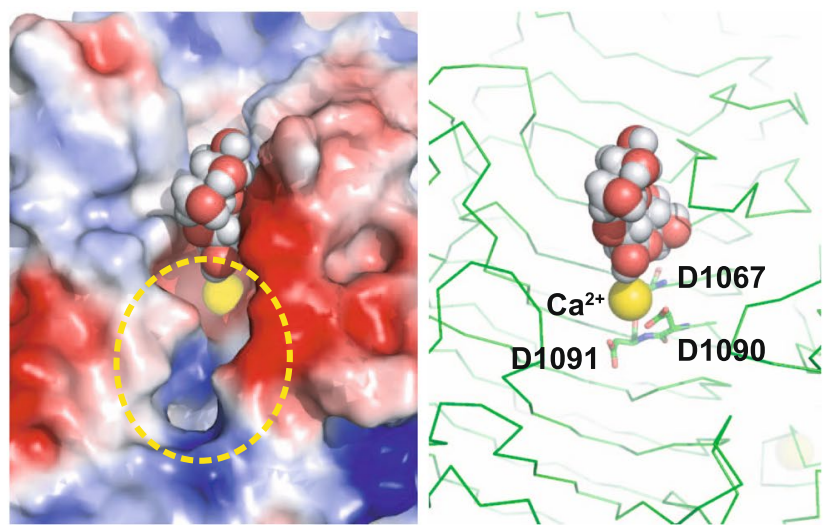

Figure 4. (a) Positions of D1067, D1090 and D1091. These three residues are shown in red. The galactosebinding-like domain is shown in blue. The enlarged view is shown at right. (b) Docking simulation results using a nigerose molecule. Left: the nigerose molecule is shown with sphere. Right: the glutamate triad is shown with stick representation, and $\mathrm{Ca}^{2+}$ is shown with a yellow sphere. Protein $\mathrm{C} \alpha$ trace is shown with green line. Substrate-binding pocket is indicated with a dashed yellow circle.

with the galactose-binding-like domain and the secondary structure topology is also different. Despite the proposal that the GH 49 and GH 87 families share a common evolutionary ancestor ${ }^{18}$, these complex structures seem to be independently acquired during evolution.

The $\beta$-helix consists of 12 turns, with 24-38 amino acids per turn (Fig. 3e). According to the original definition of a $\beta$-helix by Yoder et al., one $\beta$-helix consists of three $\beta$-strands, PB1, PB2 and PB3, connected via three turns, $\mathrm{T} 1, \mathrm{~T} 2$ and $\mathrm{T} 3^{20}$. The $\mathrm{N}$ - and $\mathrm{C}$-terminal coils are considered incomplete coils comprising $\mathrm{PB} 2$ and PB3 for the $\mathrm{N}$-terminal coil and PB1 and PB2 for the $\mathrm{C}$-terminal coil. This is commonly observed as inter-strand aromatic stacking and an asparagine ladder in the $\beta$-helix fold ${ }^{20}$; such ladders are observed at the C-terminal $\beta$-helix of Agl $\triangle$ DCD-UCD (Fig. 3c). Four phenylalanine residues (F1168, F1204, F1230 and F1268) are stacked inside the PB2, and three asparagine residues (N1208, N1232 and N1270) are aligned at the coils of 8, 9, 10 and 11. Isoleucine residues are also aligned at $\mathrm{PB} 1$, which is at the opposite side of the phenylalanine ladder. The $\beta$-helix fold creates a concave surface along with the $\beta$-helix direction, resulting in a consecutive groove of the putative substrate recognition cleft (Fig. 3c,d).

The crystal structure revealed the positions of mutational residues that affected enzymatic activity. Mutations of D763, E889, D1067, D1090 and D1091 led to $<50 \%$ enzyme activity after 30 min of incubation. Among these residues, D763 and E889 were located in the galactose-binding-like domain at the interface with the $\beta$-helix domain; these are not likely to be involved in the enzymatic reaction directly because these are locate relatively far from the putative substrate-binding pocket (Supplementary Fig. S4). Rather, these positions may be important for complex formation via domain-domain interactions. In particular, E889 forms an ionic pair with R1060 of the $\beta$-helix domain (Supplementary Fig. S4). Thus, mutations in these two residues may result in the destabilisation of the complex. Mutations of D1067, D1090 and D1091 drastically reduced enzymatic activity, particularly with alanine and asparagine substitutions (Table 1). Interestingly, these three aspartate residues are conserved among the GH $28^{21-23}$ and GH $49^{19}$ families and appear to form the active site. Based on sequence similarity, these residues were predicted to also serve as the putative catalytic site for the $\mathrm{GH} 87$ family $^{18}$. These three residues are located at the very centre of the putative active cleft (Fig. 4a). Interestingly, a $\mathrm{Ca}^{2+}$ ion is coordinated to the carboxy group 
of D1067, D1090 and water molecules, with pentagonal-bipyramidal coordination. A similar $\mathrm{Ca}^{2+}$ ion coordination on the $\beta$-helix was observed in polysaccharide lyase, contributing to polysaccharide binding and enzymatic activity ${ }^{24,25}$. Thus, $\mathrm{Ca}^{2+}$ at the catalytic site of Agl $\triangle \mathrm{DCD}-\mathrm{UCD}$ may have a similar role in the reaction.

At the centre of the substrate-binding cleft, a loop from the galactose-binding-like domain appears to be protruding close to the D1067, D1090 and D1091 sites (Fig. 4a). Although the role of the loop of the galactose-binding-like domain remains unclear, this interesting structural feature may contribute to $\alpha$-1,3-glucane recognition via its saccharide-binding ability.

Docking simulation using nigerose. To evaluate the substrate binding of Agl $\triangle \mathrm{DCD}-\mathrm{UCD}$, we performed docking simulation using a nigerose molecule, a disaccharide molecule with two glucose molecules linked via a $\alpha-1,3$ linkage as the ligand. One of the solutions showed positioning at the substrate-binding cleft near D1067, D1090 and D1091 (Fig. 4b). Although docking is a rough estimation of the binding mode, it at least represents a possible binding structure. The putative- 1 subsite is located onto the carboxy group of D1067. According to the reaction mechanism of the GH 28 and GH 49 families, the cleavage of the $\alpha-1,3$ linkage may occur by the inverting mechanism with acid-base catalysis at this position.

A binding pocket (dashed yellow circle, Fig. 4b) is located on the opposite side of the docked nigerose molecule, with the size of a dimer-to-tetramer saccharide molecule. Thus, it is feasible to hydrolyse the $\alpha-1,3$ covalent bond of $\alpha-1,3$-glucan at the dimer-to-tetramer length. Indeed, Agl-KA dominantly produces tetrasaccharide, and disaccharide is also released ${ }^{26}$. This structure strikingly explains the molecular basis of the enzyme reaction. We hypothesise that the binding subsites are $(-2)(-1)(+1)(+2)(+3)(+4)$ and propose a reaction mechanism as follows: (I) $\alpha-1,3$-glucan binds to the binding cleft; (II) the $\alpha-1,3$-glucan is hydrolysed and a nick is formed; (III) the processed $\alpha-1,3$-glucan is translocated to the end of the pocket and is hydrolysed; and (IV) the tetrasaccharide is released from the pocket.

In a previous study, we have reported the x-ray crystallographic analysis of the catalytic unit of Q-1,3-glucananse AglFH1 from Paenibacillus glycanilyticus FH11 (approximately 20\% identity with Agl $\triangle$ DCD-UCD), which was prepared using a Brevibacillus expression system ${ }^{27}$. The crystal structure of the catalytic unit of AglFH1 was determined by the Native-SAD method, and crystallographic analysis of the complexes of AglFH1 with dimeric, trimeric or tetrameric saccharides of $\alpha$-1,3-glucan are currently underway. These results will provide information on the substrate-binding pocket and insights into the hydrolysing mechanism of $\alpha-1,3$-glucan based on comparison with the results of Agl $\triangle \mathrm{DCD}-\mathrm{UCD}$.

\section{Conclusions}

Here, we describe the novel structural feature of the C-terminal catalytic unit of GH 87-type $\alpha$-1,3-glucanase from $B$. circulans KA-304. The enzyme structure explains the molecular mechanism of the reaction product because of the size of the reaction pocket. Because of the scarcity of $\alpha-1,3$-glucan, the biochemical analysis of $\alpha$-1,3-glucanase represents potential difficulty. In this regard, structural analysis plays an important role for complementing the limit of the biochemical assay. The accumulating structural information of the GH 87 family enzymes, in addition to the knowledge of the substrate complex structure, should reveal the precise molecular mechanism of the enzymatic reaction in the future. Detailed understanding of the GH 87-type $\alpha$-1,3-glucanase will also expand the industrial applications of this enzyme, such as in antifungal drugs, by enabling its structure-based engineering.

\section{Materials and Methods}

Microorganisms and culture. E. coli $\mathrm{DH} 5 \alpha$ cells were used as a host to construct various recombinant plasmids grown at $37^{\circ} \mathrm{C}$ while shaking $(100 \mathrm{rpm})$ in $\mathrm{LB}$ medium containing $100 \mu \mathrm{g} / \mathrm{mL}$ ampicillin. E. coli Rosettagami B (DE3), which harboured a recombinant plasmid, was grown at $30^{\circ} \mathrm{C}$ while shaking $(100 \mathrm{rpm})$ in $\mathrm{LB}$ medium containing $100 \mu \mathrm{g} / \mathrm{mL}$ ampicillin, $10 \mu \mathrm{g} / \mathrm{mL}$ chloramphenicol, $25 \mu \mathrm{g} / \mathrm{mL}$ kanamycin and $15 \mu \mathrm{g} / \mathrm{mL}$ tetracycline.

Site-direct mutagenesis. The previously constructed pET-Agl $\triangle \mathrm{DCD}-\mathrm{UCD}$ plasmid was used as a template to generate mutants of the catalytic unit of Agl-KA. All mutant plasmids were generated using QuikChange methods (Agilent Technologies) ${ }^{28}$. PCR was performed under the following conditions: one cycle of $94^{\circ} \mathrm{C}$ for $2 \mathrm{~min}$, followed by 18 cycles of $98^{\circ} \mathrm{C}$ for $10 \mathrm{~s}, 55^{\circ} \mathrm{C}$ for $5 \mathrm{~s}$ and $72^{\circ} \mathrm{C}$ for $7.5 \mathrm{~min}$. Sequences of the mutagenic primers are listed in Supplementary Table S1. PCR products were treated with $D p n \mathrm{I}$ at $37^{\circ} \mathrm{C}$ for $1 \mathrm{~h}$ to digest the methylated template and then transformed into E. coli JM 109. Mutant plasmids were collected and sequenced to confirm the desired mutation.

Enzyme production and purification. All mutant plasmids were transformed into E. coli Rosetta-gami $\mathrm{B}$ (DE3) for expression. The transformants were cultured at $30^{\circ} \mathrm{C}$ in LB medium. When the optical density at $600 \mathrm{~nm}$ reached $\sim 0.6$, isopropyl- $\beta$-D-thiogalactopyranoside was added to the culture medium at a final concentration of $0.4 \mathrm{mM}$. Cultures were incubated further for $12 \mathrm{~h}$. E. coli cells harbouring expression plasmids were harvested and disrupted by sonication $(10 \mathrm{~min}, 350-400 \mu \mathrm{A})$ on ice. Agl $\triangle \mathrm{DCD}-\mathrm{UCD}$ and its mutants were purified according to the method previously described ${ }^{13}$.

Concentrations of Agl $\triangle \mathrm{DCD}-\mathrm{UCD}$ and mutants were estimated by measuring absorbance at $280 \mathrm{~nm}$ with the molar absorption coefficients $\left(154.130 \mathrm{M}^{-1} \mathrm{~cm}^{-1}\right)$, calculated on the basis of their amino acid compositions ${ }^{29}$. SDS-PAGE was performed using the method of Laemmli ${ }^{30}$. Pre-stained Protein Markers Broad Range (Nacalai Tesque, Kyoto, Japan) was used as a molecular marker.

$\alpha-1,3-G l u c a n a s e$ activity assay. The reaction solution containing $1 \% \alpha-1,3$-glucan, $50 \mathrm{mM}$ potassium phosphate buffer ( $\mathrm{pH} 6.5$ ), and appropriate enzyme concentrations was incubated at $30^{\circ} \mathrm{C}$. The reaction was 
quenched by placing the sample at $100^{\circ} \mathrm{C}$ for $15 \mathrm{~min}$. The suspension was centrifuged, and the precipitated $\alpha-1,3$-glucan was removed. The amount of the reducing sugars in the supernatant was determined using dinitrosalicylic acid according to the method of Miller ${ }^{31}$.

Chemical modification of carboxyl groups. The reaction mixture containing $3 \mathrm{nmol} / \mathrm{mL}$ of $\mathrm{Agl} \triangle \mathrm{DCD}-\mathrm{UCD}, 50 \mathrm{mM} \mathrm{MES} / \mathrm{NaOH}(\mathrm{pH} 5.5)$ and various concentrations of EDC was incubated at $25^{\circ} \mathrm{C}$. After incubation for a given period $(20,40$ and $60 \mathrm{~min}), 10 \mu \mathrm{L}$ of the reaction mixture was withdrawn and added to $40 \mu \mathrm{L}$ of $100 \mathrm{mM}$ MES/NaOH ( $\mathrm{pH}$ 5.5) to quench the residual reagent. The residual activity of the diluted reaction mixture was then determined.

CD measurement. The CD spectra of the purified wild-type and mutant enzymes $(0.05 \mathrm{mg} / \mathrm{mL})$ were measured at $25^{\circ} \mathrm{C}$ using a spectropolarimeter (JASCO model J-820, cell light 1-cm) in the far-UV region (200$280 \mathrm{~nm}$ ). Background was corrected against $10 \mathrm{mM}$ potassium phosphate buffer ( $\mathrm{pH} 6.5)$. The other conditions of the CD spectra were as follows: data interval, $0.5 \mathrm{~nm}$; scan speed, $100 \mathrm{~nm} / \mathrm{min}$; accumulation times, 3; band width, $1.0 \mathrm{~nm}$ and sensitivity, $100 \mathrm{mdeg}$.

ICP-MS analysis for $\mathrm{Ca}^{2+}$ and $\mathrm{Zn}^{2+}$ detection. ICP-MS was used for metal-ion identification. The purified enzyme was dialysed against deionised water and subsequently treated with $0.1 \mathrm{M} \mathrm{HNO}_{3}$. Samples were analysed in triplicate runs on an ICP-MS system (ELAN DRC II, Perkin Elmer Co.). Total $\mathrm{Ca}^{2+}$ and $\mathrm{Zn}^{2+}$ concentrations were measured using an external calibration curve determined with reference standards for each ion.

Crystal structure determination. The selenomethionine derivative of Agl-KA-cat was expressed in the B834(DE3) strain using M9 medium with $0.0025 \%$ selenomethionine and $0.04 \%$ of an amino acid mix containing lysine, leucine, isoleucine, threonine, phenylalanine and valine. Native and selenomethionine derivative crystals were obtained using the hanging-drop vapour diffusion method with crystallisation buffers containing $10 \%-13 \%$ PEG6000, $10 \mathrm{mM} \mathrm{ZnSO}_{4}$ and $0.1 \mathrm{M}$ HEPES pH 8.5 at $20^{\circ} \mathrm{C}$. The obtained crystals were then soaked in the crystallisation buffer with $30 \%$ PEG400 as a cryoprotectant and flash cooled in liquid nitrogen. Crystals were stored until the diffraction measurement.

Synchrotron X-ray diffraction measurements were performed at the beamline BL5A of Photon Factory, Tsukuba, Japan. Crystals belonged to the space group P2 $2_{1}$. MAD data were collected at the absorption peak $(0.97911 \AA)$, edge $(0.97922 \AA)$ and remote peak $(0.96400 \AA)$. The MAD and native datasets were collected at resolutions $2.0 \AA$ and $1.83 \AA$, respectively. Data were indexed and integrated using the $\mathrm{xds}^{32}$ programmes. The initial phase was determined by the Se-MAD method with the programme Phenix.autosol ${ }^{33}$. The initial protein model determined by the SeMet MAD phasing was used; further refinement and model building were performed using the native dataset in Phenix.refine ${ }^{33}$ and $\operatorname{coot}^{34}$. Data collection and refinement statistics are shown in Table 2. The structural data were deposited to protein data bank through PDBj (https://pdbj.org); the assigned PDBID is 5ZRU. Molecular structures were depicted with PyMol (https://pymol.org/). Electrostatic potential was calculated using the APBS tool (Adaptive Poisson-Boltzmann Solver). Conserved amino acids in the structure were depicted using Consurf ${ }^{35}$. Interface calculations were performed using PDBePISA (http://www.ebi.ac.uk/pdbe/prot_int/ pistart.html) ${ }^{36}$.

Docking simulation. Docking simulation was performed using the SwissDock server ${ }^{37}$. The crystal structure of Agl $\triangle \mathrm{DCD}-\mathrm{UCD}$ was used as the target molecule and nigerose was used as the ligand. The molecular structure of nigerose was obtained from PubChem (https://pubchem.ncbi.nlm.nih.gov). Results were processed using UCSF Chimera ${ }^{38}$. From the docking results, the result with the highest score and nearest positions to D1067A, D1090A and D1091A in the putative binding cleft were selected.

Preparation of $\boldsymbol{\alpha - 1 , 3 - g l u c a n . ~} \alpha-1,3$-Glucan was prepared from sucrose using glucosyltransferase I (GTF-I) of Streptococcus mutans ATCC700610 as described previously ${ }^{13}$. The GTF-I-expressing plasmid was (pET-gtf1) was introduced into E. coli Rosetta-gami B (DE3). The cell free extract of E. coli cells from $5 \mathrm{~L}$ culture was used as GTF-I preparation. The GTF-I preparation and 20\% sucrose were incubated in $5 \mathrm{~L} 50 \mathrm{mM}$ potassium phosphate buffer ( $\mathrm{pH} 7$ ) at $30^{\circ} \mathrm{C}$. After $48 \mathrm{~h}$ incubation, insoluble glucans were collected by centrifugation, and the precipitate was dissolved in $500 \mathrm{~mL} 1 \mathrm{M} \mathrm{NaOH}$. The mixture was heated at $60^{\circ} \mathrm{C}$ for $20 \mathrm{~min}$, and the mixture was neutralised with $6 \mathrm{M} \mathrm{HCl}$. The neutralised mixture was added to $500 \mathrm{~mL}$ cold ethanol. After centrifugation, alcohol-precipitated glucan was washed twice with distilled water and lyophilised. The lyophilised powder was used as $\alpha$-1,3-glucan.

Received: 4 July 2019; Accepted: 8 October 2019;

Published online: 25 October 2019

\section{References}

1. Sietsma, J. H. \& Wessels, J. G. H. Chemical analysis of the hyphal walls of Schizophyllum commune. Biochim. Biophys. Acta - Gen. Subj. 496, 225-239 (1977).

2. Yakushiji, T., Inoue, M. \& Koga, T. Inter-serotype comparison of polysaccharides produced by extracellular enzymes from Streptococcus mutans. Carbohydr. Res. 127, 253-266 (1984).

3. Dekker, N. et al. Role of the $\alpha$-Glucanase Agn1p in Fission-Yeast Cell Separation. Mol. Biol. Cell 15, 3903-3914 (2004).

4. Fuglsang, C. C. et al. Biochemical analysis of recombinant fungal mutanases. A new family of alpha1,3-glucanases with novel carbohydrate-binding domains. J. Biol. Chem. 275, 2009-2018 (2000).

5. Grün, C. H. et al. Mechanism of action of the endo - $(1 \rightarrow 3)$ - $\alpha$-glucanase MutAp from the mycoparasitic fungus Trichoderma harzianum. FEBS Lett. 580, 3780-3786 (2006). 
6. Wei, H., Scherer, M., Singh, A., Liese, R. \& Fischer, R. Aspergillus nidulans $\alpha-1,3$ Glucanase (Mutanase), mutA, Is Expressed during Sexual Development and Mobilizes Mutan. Fungal Genet. Biol. 34, 217-227 (2001).

7. Hakamada, Y. et al. Nucleotide and deduced amino acid sequences of mutanase-like genes from Paenibacillus isolates: Proposal of a new family of glycoside hydrolases. Biochimie 90, 525-533 (2008).

8. Shimotsuura, I., Kigawa, H., Ohdera, M., Kuramitsu, H. K. \& Nakashima, S. Biochemical and Molecular Characterization of a Novel Type of Mutanase from Paenibacillus sp. Strain RM1: Identification of Its Mutan-Binding Domain, Essential for Degradation of Streptococcus mutans Biofilms. Appl. Environ. Microbiol. 74, 2759-2765 (2008).

9. Sumitomo, N., Saeki, K., Ozaki, K., Ito, S. \& Kobayashi, T. Mutanase from a Paenibacillus isolate: Nucleotide sequence of the gene and properties of recombinant enzymes. Biochim. Biophys. Acta - Gen. Subj. 1770, 716-724 (2007).

10. Yano, S., Yamamoto, S., Toge, T., Wakayama, M. \& Tachiki, T. Occurrence of a Specific Protein in Basidiomycete-lytic Enzyme Preparation Produced by Bacillus circulans KA-304 Inductively with a Cell-wall Preparation of Schizophyllum commune. Biosci. Biotechnol. Biochem. 67, 1976-1982 (2003).

11. Yoshimi, A. et al. Functional Analysis of the $\alpha-1,3-$ Glucan Synthase Genes agsA and agsB in Aspergillus nidulans: AgsB Is the Major Q-1,3-Glucan Synthase in This Fungus. PLoS One 8, e54893 (2013).

12. Fujikawa, T. et al. Surface $\alpha-1,3$-Glucan Facilitates Fungal Stealth Infection by Interfering with Innate Immunity in Plants. PLoS Pathog. 8, e1002882 (2012).

13. Suyotha, W. et al. Domain Structure and Function of $\alpha-1,3$-Glucanase from Bacillus circulans KA-304, an Enzyme Essential for Degrading Basidiomycete Cell Walls. Biosci. Biotechnol. Biochem. 77, 639-647 (2013).

14. Suyotha, W. et al. Characterization of $\alpha$-1,3-glucanase isozyme from Paenibacillus glycanilyticus FH11 in a new subgroup of family $87 \alpha$-1,3-glucanase. J. Biosci. Bioeng. 118, 378-385 (2014).

15. Orengo, C. A. et al. CATH - a hierarchic classification of protein domain structures. Struct. 5, 1093-1108 (1997).

16. Mitchell, A. L. et al. InterPro in 2019: Improving coverage, classification and access to protein sequence annotations. Nucleic Acids Res. 47, D351-D360 (2019).

17. Ciccarelli, F. D., Copley, R. R., Doerks, T., Russell, R. B. \& Bork, P. CASH - A $\beta$-helix domain widespread among carbohydratebinding proteins. Trends Biochem. Sci. 27, 59-62 (2002).

18. Rigden, D. J. \& Franco, O. L. $\beta$-Helical catalytic domains in glycoside hydrolase families 49, 55 and 87: Domain architecture, modelling and assignment of catalytic residues. FEBS Lett. 530, 225-232 (2002).

19. Larsson, A. M., Andersson, R., Ståhlberg, J., Kenne, L. \& Jones, T. A. Dextranase from Penicillium minioluteum: Reaction Course, Crystal Structure, and Product Complex. Structure 11,1111-1121 (2003).

20. Yoder, M. D., Lietzke, S. E. \& Jurnak, F. Unusual structural features in the parallel $\beta$-helix in pectate lyases. Structure 1, 241-251 (1993).

21. Van Santen, Y. et al. 1.68- - crystal structure of endopolygalacturonase II from Aspergillus niger and identification of active site residues by site-directed mutagenesis. J. Biol. Chem. 274, 30474-30480 (1999).

22. Pickersgill, R., Smith, D., Worboys, K. \& Jenkins, J. Crystal structure of polygalacturonase from Erwinia caratovora ssp. carotovora. J. Biol. Chem. 273, 24660-24664 (1998).

23. Federici, L. et al. Structural requirements of endopolygalacturonase for the interaction with PGIP (polygalacturonase-inhibiting protein). Proc. Natl. Acad. Sci. 98, 13425-13430 (2002).

24. Close, D. W., D’Angelo, S. \& Bradbury, A. R. M. A new family of $\beta$-helix proteins with similarities to the polysaccharide lyases. Acta Crystallogr. Sect. D Biol. Crystallogr. 70, 2583-2592 (2014).

25. Jenkins, J., Shevchik, V. E., Hugouvieux-Cotte-Pattat, N. \& Pickersgill, R. W. The Crystal Structure of Pectate Lyase Pel9A from Erwinia chrysanthemi. J. Biol. Chem. 279, 9139-9145 (2004).

26. Yano, S. et al. Deletion of uncharacterized domain from $\alpha$-1,3-glucanase of Bacillus circulans KA-304 enhances heterologous enzyme production in Escherichia coli. J. Gen. Appl. Microbiol, https://doi.org/10.2323/jgam.2017.12.005 (2018).

27. Intuy, R. et al. X-ray crystallographic analysis of the catalytic domain of $\alpha$-1,3-glucanase FH1 from Paenibacillus glycanilyticus overexpressed in Brevibacillus choshinensis. Acta Crystallogr. Sect. F Struct. Biol. Commun. 74, 770-773 (2018)

28. Liu, H. \& Naismith, J. H. An efficient one-step site-directed deletion, insertion, single and multiple-site plasmid mutagenesis protocol. BMC Biotechnol. 8, 91 (2008).

29. Pace, C. N., Vajdos, F., Fee, L., Grimsley, G. \& Gray, T. How to measure and predict the molar absorption coefficient of a protein. Protein Sci. 4, 2411-2423 (1995).

30. Laemmli, U. K. Cleavage of Structural Proteins during the Assembly of the Head of Bacteriophage T4. Nature 227, 680-685 (1970).

31. Miller, G. L. Use of Dinitrosalicylic Acid Reagent for Determination of Reducing Sugar. Anal. Chem. 31, 426-428 (1959).

32. Kabsch, W. XDS. Acta Crystallogr. Sect. D Biol. Crystallogr. 66, 125-132 (2010).

33. Adams, P. D. et al. PHENIX: A comprehensive Python-based system for macromolecular structure solution. Acta Crystallogr. Sect. D Biol. Crystallogr. 66, 213-221 (2010).

34. Emsley, P. \& Cowtan, K. Coot: Model-building tools for molecular graphics. Acta Crystallogr. Sect. D Biol. Crystallogr. 60, $2126-2132$ (2004).

35. Ashkenazy, H. et al. ConSurf 2016: an improved methodology to estimate and visualize evolutionary conservation in macromolecules. Nucleic Acids Res. 44, W344-W350 (2016).

36. Krissinel, E. \& Henrick, K. Inference of Macromolecular Assemblies from Crystalline State. J. Mol. Biol. 372, 774-797 (2007).

37. Grosdidier, A., Zoete, V. \& Michielin, O. SwissDock, a protein-small molecule docking web service based on EADock DSS. Nucleic Acids Res. 39, 270-277 (2011).

38. Pettersen, E. F. et al. UCSF Chimera-a visualization system for exploratory research and analysis. J. Comput. Chem. 25, 1605-1612 (2004).

\section{Acknowledgements}

We thank the beamline staff at KEK-PF, Tsukuba, Japan, for their assistances. We are also grateful to Dr. Atsushi Sasaki (Yamagata University) for operating ICP-MS. This work was supported in part by JSPS KAKENHI Grant Number JP 17K07708.

\section{Author contributions}

Y.S., W.S., M.W. and K.M. designed the experiments. Y.S., W.S., N.O., T.I., T.H. and K.M. performed the experiments. T.M., Y.T., S.S. and K.M. determined the crystal structure. Y.S., M.W. and K.M. wrote the manuscript.

\section{Competing interests}

The authors declare no competing interests. 


\section{Additional information}

Supplementary information is available for this paper at https://doi.org/10.1038/s41598-019-51822-5.

Correspondence and requests for materials should be addressed to K.M.

Reprints and permissions information is available at www.nature.com/reprints.

Publisher's note Springer Nature remains neutral with regard to jurisdictional claims in published maps and institutional affiliations.

(c) (i) Open Access This article is licensed under a Creative Commons Attribution 4.0 International License, which permits use, sharing, adaptation, distribution and reproduction in any medium or format, as long as you give appropriate credit to the original author(s) and the source, provide a link to the Creative Commons license, and indicate if changes were made. The images or other third party material in this article are included in the article's Creative Commons license, unless indicated otherwise in a credit line to the material. If material is not included in the article's Creative Commons license and your intended use is not permitted by statutory regulation or exceeds the permitted use, you will need to obtain permission directly from the copyright holder. To view a copy of this license, visit http://creativecommons.org/licenses/by/4.0/.

(C) The Author(s) 2019 